\title{
Spatial directions and situation model organization
}

\author{
Gabriel A. RAdVAnSKY \\ University of Notre Dame, Notre Dame, Indiana
}

\begin{abstract}
Do spatial directions, such as "to the right," influence the integration and segregation of information into situation models? According to a single-framework hypothesis, spatial location serves as an event framework, and spatial directions serve as relational information within that framework but do not establish separate sublocation frameworks. Alternatively, according to a fragmented-framework hypothesis, spatial directions lead the larger framework to be broken down such that each direction is treated as a separate sublocation, thereby producing retrieval interference. In three experiments, people memorized sentences about objects in locations. The results support the fragmented-framework hypothesis. Control conditions ruled out explanations based on the ease of memorization, retrieval demands, or sentence complexity.
\end{abstract}

When people encounter a spatial direction within a larger spatial framework, such as "to the left in the library," how is the directional information handled? That is, if language serves as instructions for creating a situation model (Zwaan, 1999), what impact do spatial directions have on this process? A situation model is a mental representation of a described state of affairs that serves as a mental simulation of those events (e.g., Zwaan \& Radvansky, 1998). Successful comprehension requires not only an adequate processing of the language itself, but also the creation of an appropriate representation of the events being described (Glenberg, Meyer, \& Lindem, 1987), and language serves as a set of instructions for how to create these situation models (Zwaan, 1999). As such, it is important to understand how different types of language - spatial directions in this case-influence how these situation models are eventually constructed and organized.

In general, the spatial information that can be used to structure a situation model can be classified in two ways: spatial frameworks and spatial relations (Wyer \& Radvansky, 1999). Spatial framework information is the region that serves as the bounds of an event, such as a building, room, or any well-defined, labeled area, such as park. In some sense, this is absolute spatial knowledge. The other type of spatial knowledge is spatial relational information. This is information that denotes the spatial interrelations of entities with respect to each other, such as something being to the right, left, above, and so on. So, in some sense, this is relative spatial knowledge.

These different types of spatial knowledge are thought to be captured by different aspects of situation models (Wyer \& Radvansky, 1999). The spatial framework information establishes the boundaries of the event - the context in which the event unfolds. Spatial framework information is a basis of organizing situational information in memory (e.g., Radvansky, 1999a), influencing the access of knowledge about events, both in text (e.g., Morrow, Greenspan, \& Bower, 1987) and in interactive situations (e.g., Radvansky \& Copeland, 2006c), and shifts in spatial frameworks can disrupt reading (e.g., Zwaan, Radvansky, Hilliard, \& Curiel, 1998).

In comparison, spatial relations capture the interrelations among entities within the spatial-temporal framework. These spatial relations convey the internal structure of elements within the framework. Spatial relation information can influence the availability of information from situation models. For example, information is differentially available along different internal axes (Franklin \& Tversky, 1990) and is influenced by the presence or absence of a functional relation between entities (Radvansky \& Copeland, 2000).

This distinction between spatial framework and relational information parallels other work in the literature. For example, there is the difference between metric and categorical spatial knowledge (e.g., Huttenlocher, Hedges, \& Duncan, 1991); the difference between intrinsic, relative, and absolute reference frames (e.g., Levinson, 2003); as well as neurological evidence for processing these different types of information (e.g., Kemmerer, 2006; Kosslyn, 1987). Thus, there is evidence beyond the work in event cognition to suggest that spatial frameworks and relations capture psychologically different types of information.

Although we can identify and discriminate between these different types of spatial knowledge, it is not necessarily the case that they are treated distinctly in terms of the type of information that they provide about the structure and organization of the situation models that are created to capture simple ideas. Specifically, is spatial relation information only treated as providing structure about the

G. A. Radvansky, gradvans@nd.edu 
relation among items within a spatial framework or does it also play a role in defining sublocations within the larger spatial region? For example, if a person is told that one object is to the left and another is to the right, is each sublocation treated as a new mini-framework around which a situation model can then be organized? If so, one would expect this substructure to have influences that parallel those with information that is more clearly framework information, such as the name of a location.

\section{Retrieval Interference}

To assess the impact of spatial framework and relation information, in this study, we used a memory retrieval paradigm - specifically, memory for sentences. When presented with lists of sentences to learn, people create situation models of the events that they describe (Johnson-Laird, 1983; van Dijk \& Kintsch, 1983; Zwaan \& Radvansky, 1998) and use those models to make later memory decisions (e.g., Bransford, Barclay, \& Franks, 1972; Garnham, 1981; Radvansky, 1999a; Radvansky \& Zacks, 1997). One means of organizing and structuring information into situation models is on the basis of spatial locations. This sort of event model organization can be tested using a fan effect paradigm.

A fan effect (e.g., Anderson, 1974) is a response time increase accompanying an increase in the number of associations with a concept. The fan effect can be attenuated or eliminated if a set of facts is related thematically (Moeser, 1979; Myers, O'Brien, Balota, \& Toyofuku, 1984; Smith, Adams, \& Schorr, 1978) or situationally (e.g., Radvansky, 1998; Radvansky, Spieler, \& Zacks, 1993; Radvansky \& Zacks, 1991). Of specific concern here is a paradigm originally developed by Radvansky and Zacks (1991; Radvansky et al., 1993).

In this paradigm, people are given a series of facts to learn, such as sentences about objects in locations. A sample subset would be Sentences $1-6$ below.

1. The closet door is in the car dealership.

2. The coat rack is in the car dealership.

3. The fire extinguisher is in the car dealership.

4. The drinking fountain is in the city hall.

5. The drinking fountain is in the grand hotel.

6 . The drinking fountain is in the airport.

In Sentences $1-3$, there is a collection of objects all in the same place. As such, this is referred to as the single- location condition. In this case, a situation model view suggests that these sentences can be easily interpreted as referring to a single state of affairs. This information can then be integrated into a single model in memory. Later, during retrieval, there is no interference for any one of those facts (as evidence by an absence of a fan effect), since they are all part of the same mental representation.

In contrast, for Sentences 4-6, there is a single object in several different places. As such, this is referred to as the multiple-locations condition. In this case, according to a situation model view, when several facts share a common concept but are not consistent with a single situation, each one of these is represented by a separate model. As a result, during retrieval, accessing any one of these representations can result in interference. The more related but irrelevant models there are, the greater is the amount of interference. So, a fan effect is observed under these conditions.

This pattern of a fan effect for multiple-situation, but not single-situation conditions is well established (Radvansky, 1999a, 1999b, 2005). It occurs despite instructions to organize by other means (Radvansky, 1998) — with definite or indefinite articles (e.g., the vs. a/an) (Radvansky et al., 1993), when the location serves as either the sentence subject or predicate (e.g., "the potted palm is in the hotel" vs. "in the hotel is the potted palm"; Radvansky \& Zacks, 1991), with both text and picture stimuli (Radvansky \& Copeland, 2006b), and in both younger and older adults (Radvansky, Zacks, \& Hasher, 1996, 2005). It is not strictly a spatial effect, in that it has also been observed with ownership (Radvansky, Wyer, Curiel, \& Lutz, 1997) and temporal relations (Radvansky, Zwaan, Federico, \& Franklin, 1998). Basically, integrated representations do not produce a fan effect, whereas separately stored but related representations do (e.g., Moeser, 1979; Smith et al., 1978).

\section{Theoretical Alternatives}

Now that the types of spatial information and the experimental paradigm have been outlined, the predictions can be made. An illustration of the two positions is shown in Figure 1. According to a single-framework hypothesis, the role of spatial directions is to provide information about the structure of a situation within the context of a larger spatial framework. That is, saying that something is "to the right" does not define a new location, but only provides detail about the single, larger location. It does not take on

\section{Single Framework}

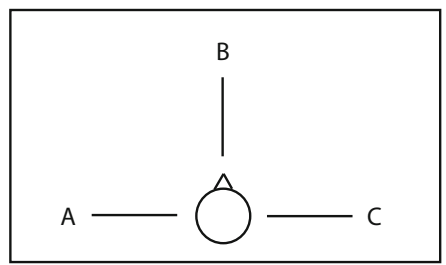

Fragmented Framework

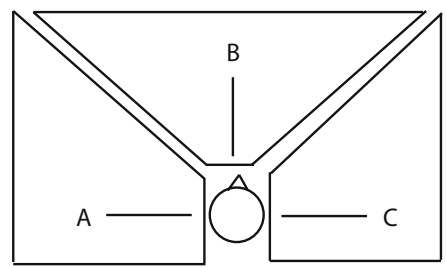

Figure 1. An illustration of the basic concepts behind the single-framework and fragmented-framework views of situation model organization for spatial direction terms. 
the role of further subdividing the framework. As such, the inclusion of direction information would not influence the organization of information into situation models and would have no impact on memory retrieval beyond that already observed with spatial framework information. The directions only provide extra detailed information about the objects and their interrelations. So, the prediction for a single-framework view is that, like those studies in which direction information was not included, there will be a fan effect for the multiple-locations condition but not for the single-location condition.

In comparison, according to a fragmented-framework hypothesis, spatial directions subdivide a larger spatial framework into subregions. In other words, spatial directions not only provide information about the relative position of objects with respect to one another, but also serve to define different frames of reference. So, saying that something is "to the right" establishes a new location framework within the context of the larger spatial framework, such as a building or room. The prediction for a fragmented-framework view is that there will be a fan effect for both the single-location and the multiple-locations conditions. The degree of the interference in each of these cases is unclear. On one hand, the level of interference could be the same if the sublocations are treated as completely distinct representations during memory retrieval. On the other hand, the sublocations are defined with respect to the larger framework. As such, this confines the search of memory, thereby limiting the amount of interference experienced. So, there would be a fan effect in the single-location condition, although it would not be as great as in the multiple-locations condition.

So, in sum, in these experiments, some of the study sentences described multiple objects in single locations, and some sentences described an individual object in multiple locations (with spatial directions within each location for both conditions). The single-framework hypothesis suggests that people will create a model of the single location that is not subdivided on the basis of the spatial terms. Thus, the single location should continue to function as a single location and should produce no fan effect. In contrast, the fragmented-framework hypothesis suggests that the single location is broken down into several submodels, consistent with the spatial terms. Thus, the single location should now function as multiple locations and should produce a fan effect.

\section{EXPERIMENT 1}

In Experiment 1, people were given sets of facts to learn about objects in locations. In addition, the study facts contained spatial relations about the relative location of various objects (e.g., "the potted palm is to the left at the hotel"), with each particular direction term occurring only once for a given object or location. According to the single-framework hypothesis, there will be a differential fan effect, with a clear interference effect in the multiplelocations condition but not in the single-location condition. Alternatively, according to a fragmented-framework hypothesis, the prediction is that there will be less integration, and there would be interference in the single-location condition, as well as in the multiple-locations condition.

In addition, for Experiment 1, two groups of people were tested. In one group (complete probes), the recognition probes contained the object, location, and direction, as in the study sentences. This assessed performance when spatial directions were present at both learning and test. In the other group (abbreviated probes), people received recognition probes that had the object and location terms but not the direction (e.g., "the potted palm is in the hotel"). This was done to assess whether the direction terms influenced retrieval when they were not required for a correct response. It is possible that any impact of directions with the complete probes could be a result of the need to retrieve direction information per se. As such, by removing this need, any impact of directions on the observed pattern of data can be more confidently attributed to the underlying organization in memory, rather than to task demands at retrieval. Finally, it should be noted that previous studies (e.g., Radvansky, 1999a) did not include direction terms at all. So, these abbreviated recognition probes more closely paralleled that work.

\section{Method}

Participants. Fifty-one participants were recruited from the participant pool at the University of Illinois, Urbana-Champaign, and were given partial course credit for their participation. Twenty-four of these participants were in the abbreviated probe group, and the rest were in the complete probe group.

Materials. People memorized a list of 18 sentences of the form "The object is direction in the location." The creation of the study lists largely followed the design outlined by Radvansky et al. (1993). The associate structure of the study list design is shown in Figure 2 for a hypothetical participant. Each participant's study list was generated through random pairings of objects and locations, so that there were 1-3 associations with each object and location concept (thus defining Fan Levels 1-3). The conditions of interest are those in which either a single object is associated with several locations (multiple-locations condition) or a single location is associated with several objects (single-location condition). To provide the appropriate number of associations for the critical items, the study lists included 2-3, 3-2, and 3-3 fan sentences $(X-Y$ refers to $X$ number of associations with the object and $Y$ number of associations with the location). There were four sentences in which both the object and the location concept had only one association each (Fan Level 1). Two of these sentences were arbitrarily assigned to the single-location condition and two to the multiple-locations condition. This was done so that the same data would not be used for Fan Level 1 in both conditions, and there would be the same number of observations in these cells as in the higher fan level cells. The objects and locations used in Experiments 1 and 2 are given in the Appendix.

In addition to the objects and locations, each sentence contained a phrase that referred to the spatial direction that the object was in. These phrases were "to the right," "to the left," and "straight ahead." The participants were told that these directions referred to where the object would be when someone first walked into the location. The occurrence of each direction term was counterbalanced throughout the study list, so that each term occurred equally often. Moreover, no direction term was repeated for any given object or location concept. Finally, the direction terms were cycled across participants, so that a given direction did not appear in the same condition for all of the participants. Instead, each direction occurred equally often in each condition across participants. 


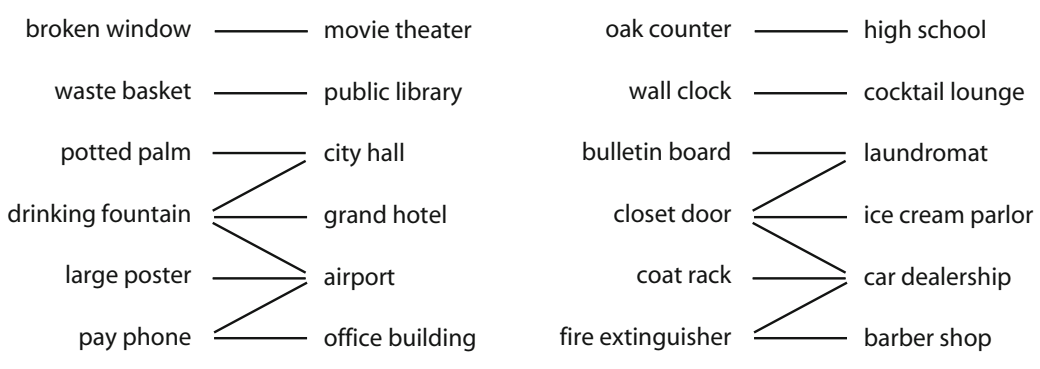

Figure 2. An example of the structure of object-location associations in the construction of the study list. Note that the positions of the object and location concepts within this structure were randomized for each participant.

For recognition, for the complete-probes groups, the probes contained the object, direction, and location. Nonstudied probes were generated from repairings of objects and locations from within the same cell of the design. For this experiment, the direction term was yoked to the location. For example, if the studied sentences from the same cell were Sentences 7 and 8 , the nonstudied sentences would be Sentences 9 and 10 .

7. The broken window is to the right in the movie theater.

8. The oak counter is straight ahead in the high school.

9. The broken window is straight ahead in the high school.

10. The oak counter is to the right in the movie theater.

This method of generating nonstudied probes avoids the possibility that the participants will use plausibility judgments (Reder \& Anderson, 1980). Because the same numbers of associations were for the nonstudied sentences, they were assigned to the single-location and multiple-locations conditions and were analyzed as such. For the abbreviated-probes group, the same sentences were used during recognition, except that the spatial relations were removed (e.g., "The broken window is in the movie theater," rather than "The broken window is to the right in the movie theater").

Procedure. The sentences were memorized using a study-test procedure. During study, the sentences were presented one at a time on a PC with the screen in 40-column mode. The sentences were presented for $7 \mathrm{sec}$ each, in white letters on a black background. The participants were told to memorize the sentences as efficiently as possible.

During the test portion of memorization, questions of the form "Where is the object?" and "What is in the location?" were presented for each object and location, respectively. The participants typed their answers into the computer and needed to include the direction term. For example, for the sentence "The potted palm is to the right in the city hall," the answer to the question "Where is the potted palm?" would be "To the right in the city hall," and the answer to the question "What is in the city hall?" would be "The potted palm is to the right." For some questions, there was more than one answer, and the participants were to provide all of the answers. A number was displayed with each question indicating how many answers there were (one, two, or three). After an answer was given, the computer indicated whether it was correct. If any incorrect answers were given, the correct answers were displayed for $3 \mathrm{sec}$ for each answer. At the end of the test portion, the computer reported how many questions were answered correctly. It then returned to the study portion. This study-test procedure continued until all correct answers were provided for two complete sets of questions.

After the sentences were memorized, the recognition test was given. During recognition, the participants were presented with 432 sentences. They indicated that the probe sentences were true by pushing the left button on a computer mouse, which was marked with a "Y" for yes, this sentence is true and indicated false sentences by pushing the right button, which was marked with an " $\mathrm{N}$ " for no, this sentence is not true. The participants were encouraged to re- spond as fast and as accurately as possible. If an incorrect response was made, the computer beeped and presented either "***EROR! SENTENCE TRUE!**” or “**ERROR! SENTENCE NOT TRUE!**," whichever was appropriate, for $1 \mathrm{sec}$. A self-timed break was allowed after every 108 trials.

To familiarize the participants with the recognition test procedure, an 18-trial practice period was given. During this period, the computer displayed a line that read either "SENTENCE TRUE" or "SENTENCE NOT TRUE," and the participants responded accordingly.

\section{Results}

Errors were trials that a person responded to incorrectly. These data were excluded from the response time analysis. In addition, the response time data were trimmed by first dropping extreme response times (faster than $500 \mathrm{msec}$ or slower than $10,000 \mathrm{msec}$ ). Following this, any response times that were more that 2.5 standard deviations from the cell mean were excluded. This trimming procedure eliminated $3.6 \%$ of the data.

Learning. The participants took two to eight study-test cycles to memorize the study sentences $(M=5.1, S E=$ $0.21)$.

Recognition. The response time and error rate data are summarized in Table 1 and were each submitted to 2 (group: complete vs. abbreviated probes) $\times 2$ (truth value: true vs. false) $\times 2$ (location: single location vs. multiple locations) $\times 3$ (fan) repeated measures ANOVA. There was a significant main effect of truth value $[F(1,49)=13.62$, $\left.M S_{\mathrm{e}}=46,872, p=.001\right]$, with the participants responding faster to true probes $(M=1,744 \mathrm{msec})$ than to false probes $(M=1,805 \mathrm{msec})$. More important, there were significant effects of location $\left[F(1,49)=52.66, M S_{\mathrm{e}}=89,920\right.$, $p<.001]$ and fan $\left[F(2,98)=33.17, M S_{\mathrm{e}}=92,430, p<\right.$ $.001]$, as well as a significant location $\times$ fan interaction $\left[F(2,98)=9.19, M S_{\mathrm{e}}=78,289, p<.001\right]$. Simple effects tests revealed that the fan effect was significant for both the single-location [Fan Level 1, $M=1,610 \mathrm{msec}$; Fan Level 2, $M=1,719$ msec; Fan Level 3, $M=1,725 \mathrm{msec} ; F(2,98)=$ 5.07, $\left.M S_{\mathrm{e}}=81,031, p=.008\right]$ and the multiple-locations conditions [Fan Level 1, $M=1,653 \mathrm{msec}$; Fan Level 2, $M=$ 1,944 msec; Fan Level 3, $M=1,996 \mathrm{msec} ; F(2,98)=37.63$, $\left.M S_{\mathrm{e}}=89,688, p<.001\right]$. Thus, there were fan effects in both conditions, although it was greater for the multiplelocation probes. Overall, despite the significant interaction, the existence of a substantial fan effect in the single-location condition is more consistent with the fragmented-framework 
Table 1

Response Times (RTs, in Milliseconds) and Error Rates (Err) for Experiment 1

\begin{tabular}{|c|c|c|c|c|c|c|c|c|c|c|c|c|}
\hline & \multicolumn{6}{|c|}{ Single Location } & \multicolumn{6}{|c|}{ Multiple Locations } \\
\hline & \multicolumn{2}{|c|}{1} & \multicolumn{2}{|c|}{2} & \multicolumn{2}{|c|}{3} & \multicolumn{2}{|c|}{1} & \multicolumn{2}{|c|}{2} & \multicolumn{2}{|c|}{3} \\
\hline & RT & Err & RT & Err & RT & Err & RT & Err & RT & Err & RT & Err \\
\hline \multicolumn{13}{|c|}{ Abbreviated Probes } \\
\hline True & 1,424 & .04 & 1,447 & .03 & 1,547 & .03 & 1,468 & .03 & 1,619 & .04 & 1,687 & .05 \\
\hline False & 1,541 & .02 & 1,605 & .01 & 1,629 & .01 & 1,536 & .03 & 1,749 & .03 & 1,858 & .01 \\
\hline \multicolumn{13}{|c|}{ Complete Probes } \\
\hline True & 1,714 & .02 & 1,907 & .02 & 1,859 & .02 & 1,752 & .02 & 2,195 & .03 & 2,164 & .04 \\
\hline False & 1,733 & .02 & 1,875 & .01 & 1,833 & .01 & 1,821 & .04 & 2,156 & .02 & 2,223 & .04 \\
\hline
\end{tabular}

hypothesis. Specifically, the individual directions are treated as separate locations and, therefore, reduce the mitigation of retrieval interference brought about by having multiple objects in a single location.

There was also a main effect of group $[F(1,49)=16.44$, $\left.M S_{\mathrm{e}}=1,094,139, p<.001\right]$, not surprisingly, with the participants responding faster to the abbreviated probes $(M=$ $1,594 \mathrm{msec})$ than to the complete probes $(M=1,936 \mathrm{msec})$. There was also a significant group $\times$ location interaction $\left[F(1,49)=5.19, M S_{\mathrm{e}}=89,920, p=.03\right]$, with the effect of location being smaller for the abbreviated-probes group [single location, $M=1,532 \mathrm{msec}$; multiple locations, $M=$ $\left.1,653 \mathrm{msec} ; F(1,23)=17.40, M S_{\mathrm{e}}=60,465, p<.001\right]$, relative to the complete-probes group [single location, $M=1,820 \mathrm{msec}$; multiple locations, $M=2,052 \mathrm{msec}$; $\left.F(1,26)=37.45, M S_{\mathrm{e}}=115,977, p<.001\right]$. Finally, there was a significant group $\times$ fan interaction $[F(2,98)=3.80$, $\left.M S_{\mathrm{e}}=92,430, p<.001\right]$, with the fan effect being smaller for the abbreviated-probes group [Fan Level 1, $M=$ 1,492 msec; Fan Level 2, $M=1,605$ msec; Fan Level 3, $M=1,681 \mathrm{msec} ; F(2,46)=8.58, M S_{\mathrm{e}}=100,348, p=$ $.001]$ relative to the complete-probes group (Fan Level 1, $M=1,755 \mathrm{msec}$; Fan Level 2, $M=2,033 \mathrm{msec}$; Fan Level 3, $M=2,020 \mathrm{msec} ; F(2,52)=31.17, M S_{\mathrm{e}}=85,425$, $p<.001]$. These two interactions with group may simply be a reflection of overall retrieval speed because of the lengths of the probes in the two groups affected the size of the observed effects in these two cases. The important point is that the basic location and fan effects were present with both probe types. More important, neither the group $\times$ location $\times$ fan nor the four-way interaction was significant (both $F_{\mathrm{s}}<1$ ). Thus, although the participants' receiving either abbreviated or complete probes during recognition may have influenced the speed of responding and the size of the basic effects, the overall pattern was unchanged. This suggests that sublocations were established during learning and continued to influence retrieval, even when spatial relation information was not needed.

In general, the error rates were low, with the participants making an average of $2.5 \%$ errors. The analysis of the error rate data revealed a significant main effect of truth value $\left[F(1,49)=5.28, M S_{\mathrm{e}}=.002, p=.008\right]$, as well as a group $\times$ truth value interaction $[F(1,49)=5.28$, $\left.M S_{\mathrm{e}}=.002, p=.008\right]$. Individual group tests revealed that, for the abbreviated probes group, there were more errors for the true probes $(M=.03)$ than for the false probes $(M=.02)\left[F(1,23)=7.97, M S_{\mathrm{e}}=.002, p=.01\right]$ but no difference for the complete-probes group $(F<1)$. There was also a significant main effect of location $[F(1,49)=$ $\left.11.02, M S_{\mathrm{e}}=.001, p=.002\right]$, with more errors in the multiple-locations $(M=.03)$ than in the single-location condition $(M=.02)$.

\section{Discussion}

The results of Experiment 1 showed a differential fan effect. That is, a larger fan effect was observed in the multiple-locations condition than in the single-location condition. This pattern of data has been interpreted as consistent with an overall location-based organization of information into situation models and the use of these models at retrieval (Radvansky, 1999a). More important for the present concerns, there was also a clear interference effect in the single-location condition. This pattern of data is more consistent with the fragmented-framework hypothesis, which suggests that people use the direction terms to create sublocations within the larger spatial framework. Each of these sublocations would be treated as a separate situation and would be segregated, to some degree, in memory, thereby allowing for interference to occur during retrieval. The larger interference effect in the multiple-locations condition is a result of interference from both location and direction information, whereas the smaller interference effect in the single-location condition is a result of interference from just the direction information.

The absence of a difference between performances for the abbreviated and complete-probe groups in the recognition test suggests that the basis for the interference was established during learning. It is not a consequence of the task demands imposed during retrieval, because the direction information was not probed for in the abbreviated probes group. Instead, during learning, the direction terms impacted how the information was stored in memory.

Finally, it is also clear that the spatial frameworks defined by the location terms had a greater impact on processing than the sublocations defined by the directions. This may be because the direction terms were made with reference to a common larger location. This attenuated the impact of interference during retrieval.

\section{EXPERIMENT 2}

The aim of Experiment 2 was to further assess whether spatial directions are treated as signals to create sublocations, as the fragmented-framework hypothesis suggests, 
or whether the observed pattern of results in Experiment 1, might be due to artifacts of the learning and testing situations.

There were two primary differences introduced in Experiment 2. The first was how the sentences were learned. In Experiment 1, the study sentences were presented one at a time. As such, this may place a heavy burden on working memory if the participants are trying to integrate information about a common location or to further subdivide locations by spatial directions. Working memory capacity has an impact on the ability to learn facts in fan-effect studies (Bunting, Conway, \& Heitz, 2004; Cantor \& Engle, 1993; Radvansky \& Copeland, 2006a). In the present circumstances, the working memory load is greater, because the participants need to keep track not only of which objects are in which location, but also of the direction in which they are placed.

To address the impact of this additional working memory load, half of the participants in Experiment 2 (standardstudy group) learned the study sentences in the same manner as those in Experiment 1. However, the other half of the participants (clustered-study group) had all of the sentences that referred to a common location presented together. This may have allowed the participants to overcome the additional load of the direction terms and to better integrate the information, eliminating the single-location fan effect observed in Experiment 1 (and presumably in the standard-study group). Alternatively, it may have allowed the participants to dispense with the need to keep track of everything that goes in one location and to more easily treat each direction as a separate location. The consequence of this would be that the fan effect in the single-location condition would be larger for the clustered study group than for the standard study group.

The second methodological difference was in the construction of the negative probes. In Experiment 1, the negatives were created by binding the spatial direction with the location. This may have biased the participants against using the direction information to make their recognition decisions. This is because the decisions could be made using the object and location information alone. In contrast, in Experiment 2, for each studied item, there were three variations used to create the nonstudied item: a change in the object, a change in the direction, and a change in the location. For example, if the studied sentence was "The fire extinguisher is straight ahead in the office building," the three versions of the nonstudied sentence would be "The clock is straight ahead in the office building," "The fire extinguisher is to the right in the office building," and "The fire extinguisher is straight ahead in the library." As such, the participants would need to process the direction information to make a recognition decision. Finally, in addition to these two changes, in Experiment 2, only the complete probes were used.

According to the single-framework hypothesis, the results of Experiment 1 may reflect the heavy working memory load during learning, the task demands of the recognition test, or both, which would have masked the true nature of how the participants use spatial directions in this context. As such, if either of these factors had an impact, we would expect the interference effect in the single-location condition to be altered. In contrast, if the participants use the direction terms to further segregate information in situation models, a pattern of data consistent with the fragmented-framework hypothesis would continue to be observed.

\section{Method}

Participants. Sixty-four people were recruited from the participant pool at the University of Notre Dame and were paid for their participation.

Materials and Procedure. The materials and procedure were similar to those in Experiment 1, except that (1) the complete probes were always presented for the 432-item recognition test, (2) the 18 study sentences were presented together by common locations in the clustered-study group, and (3) the negative probes were created by altering the object, direction, and location terms. For the negative probes, the three versions of each type of negative sentence occurred equally often across the recognition test. As in Experiment 1, half of the recognition probes were positive, and half were negative probes. Finally, the object and location names were shortened somewhat, as is noted in the Appendix. This was done to speed encoding time and to reduce variability, to better allow the analyses to pick up on any changes in the pattern of response times.

\section{Results}

Learning. The participants took $2-10$ study-test cycles to memorize the study sentences $(M=5.0, S E=0.20)$. There was no difference between the learning rate for the standard-study $(M=5.0, S E=0.35)$ and that for the clustered-study group $(M=5.0, S E=0.21)(F<1)$.

Recognition. The response time and error rate data are reported in Table 2 . The trimming procedure eliminated $3.3 \%$ of the response time data. The response time and error rate data were submitted to 2 (group: individual study vs. grouped study) $\times 2$ (truth value: true vs. false) $\times 2$ (location: single location vs. multiple locations) $\times 3$ (fan) repeated measures ANOVAs.

Table 2

Response Times (RTs, in Milliseconds) and Error Rates (Err) for Experiment 2

\begin{tabular}{|c|c|c|c|c|c|c|c|c|c|c|c|c|}
\hline & \multicolumn{6}{|c|}{ Single Location } & \multicolumn{6}{|c|}{ Multiple Locations } \\
\hline & \multicolumn{2}{|c|}{1} & \multicolumn{2}{|c|}{2} & \multicolumn{2}{|c|}{3} & \multicolumn{2}{|c|}{1} & \multicolumn{2}{|c|}{2} & \multicolumn{2}{|c|}{3} \\
\hline & RT & Err & RT & Err & RT & Err & RT & Err & RT & Err & RT & Err \\
\hline \multicolumn{13}{|c|}{ Standard Study } \\
\hline True & 1,873 & .01 & 2,174 & .02 & 1,965 & .01 & 1,879 & .01 & 2,266 & .02 & 2,335 & .01 \\
\hline False & 2,270 & .02 & 2,335 & .01 & 2,579 & .01 & 2,341 & .01 & 2,787 & .02 & 2,774 & .03 \\
\hline \multicolumn{13}{|c|}{ Clustered Study } \\
\hline True & 2,142 & .01 & 2,413 & .01 & 2,415 & .01 & 2,183 & .01 & 2,613 & .01 & 2,707 & .01 \\
\hline False & 2,684 & .01 & 2,971 & .01 & 2,753 & .01 & 2,800 & .01 & 3,036 & .01 & 3,177 & .02 \\
\hline
\end{tabular}


For the response times, there was a significant main effect of group $\left[F(1,62)=10.08, M S_{\mathrm{e}}=2,333,733, p=\right.$ $.002]$, with the participants in the standard-study group responding faster $(M=2,308 \mathrm{msec})$ than those in the clustered-study group $(M=2,658 \mathrm{msec})$. There was a significant main effect of truth value $[F(1,63)=262.92$, $\left.M S_{\mathrm{e}}=162,456, p<.001\right]$, with the participants responding faster to true probes $(M=2,247 \mathrm{msec})$ than to false probes $(M=2,719 \mathrm{msec})$.

There were significant main effects of location $\left[F(1,62)=17.65, M S_{\mathrm{e}}=368,058, p<.001\right]$ and fan $\left[F(2,124)=31.12, M S_{\mathrm{e}}=275,863, p<.001\right]$, as well as a significant location $\times$ fan interaction $[F(2,124)=3.66$, $\left.M S_{\mathrm{e}}=300,725, p=.03\right]$. Simple effects tests revealed that, as in Experiment 1, the fan effect was significant for both the single-location [Fan Level 1, $M=2,242 \mathrm{msec}$; Fan Level 2, $M=2,503 \mathrm{msec}$; Fan Level 3, $M=2,428 \mathrm{msec}$; $\left.F(2,126)=7.79, M S_{\mathrm{e}}=296,053, p=.001\right]$ and multiplelocations conditions [Fan Level 1, $M=2,301 \mathrm{msec}$; Fan Level 2, $M=2,676 \mathrm{msec}$; Fan Level 3, $M=2,748 \mathrm{msec}$; $\left.F(2,126)=27.00, M S_{\mathrm{e}}=273,208, p<.001\right]$, although this effect was larger for the multiple-locations condition. Thus, as in Experiment 1, this pattern of data is more consistent with the fragmented-framework hypothesis.

Although group did not significantly enter into any other interactions (all $F \mathrm{~s}<1$ ), there was a marginally significant four-way interaction $\left[F(2,124)=2.47, M S_{\mathrm{e}}=\right.$ $191,960, p=.09]$. Looking at the pattern of data, this is attributable to theoretically minor variations in the pattern of data. Separate analyses for the standard-study and clustered-study groups revealed that the truth value $\times$ location $\times$ fan interactions were not significant in either case $\left[F(2,62)=2.28, M S_{\mathrm{e}}=155,098, p=.12\right.$, and $F<1$, respectively].

The participants made an average of $1.2 \%$ errors. The analysis of the error rate data revealed that only the location $\times$ fan $\left[F(2,124)=4.31, M S_{\mathrm{e}}=.001, p=.02\right]$ and the truth value $\times$ location $\times$ fan interactions $[F(2,124)=$ 5.56, $\left.M S_{\mathrm{e}}=.001, p=.005\right]$ were significant. Separate analyses revealed that the location $\times$ fan interaction was not significant for the true probes $(F<1)$ but was for the false probes $\left[F(2,124)=8.80, M S_{\mathrm{e}}=.001, p<.001\right]$. Simple effects analyses of the false probes showed that the fan effect was significant for the multiple-locations condition $\left[F(2,124)=7.47, M S_{\mathrm{e}}=.001, p=.001\right]$ but not for the single-location condition $[F(2,124)=1.53$, $\left.M S_{\mathrm{e}}=.001, p=.22\right]$.

\section{Discussion}

Like in Experiment 1, there was a differential interference effect, with a greater fan effect in the multiplelocations condition than in the single-location condition, suggesting a general organization by spatial location. Furthermore, there was a significant interference effect for the single-location condition. Like in Experiment 1, this is consistent with the fragmented-framework hypothesis. That is, the participants used the direction terms to subdivide their mental representations so that the different subregions within a larger location would compete with one another during retrieval. Also, like in Experiment 1, the fact that the interference effect was much greater for the multiple-locations than for the single-location condition suggests that the location information, itself, served to constrain the memory search.

Experiment 2 also demonstrated that the organization of the information in memory does not appear to be due to the heavier working memory load that was present during memorization as a result of the additional direction terms. When the participants memorized the sentences grouped together by a common location, which eased the working memory load, the pattern of data was similar to that when each sentence was viewed one at a time in a random order. Thus, this result supports the idea that the pattern observed at retrieval is more likely to be a function of how the participants interpreted this information, not of the particular task demands during memorization.

Finally, the data from Experiment 2 further support the fragmented-framework hypothesis, in that the pattern of data was relatively unchanged when the nature of the negative probes (altering the object, location, and direction) was modified to force the participants to use the direction information during retrieval (if they had not been in Experiment 1). Thus, this result supports the idea that the pattern observed at retrieval is due to how the participants treated the direction terms during memorization and was not due to task demands at retrieval.

\section{EXPERIMENT 3}

One alternative account of the results of Experiments 1 and 2 is that the patterns of data are not due to the processing of spatial information per se. Instead, it could be due to the complexity of the study sentences. That is, including extra phrases, such as "to the right," in the study sentences, created an additional source of interference, which was then observed at retrieval. As such, according to such a sentence-complexity hypothesis, the inclusion of any additional phrases to the study sentences, spatial or not, should have a similar effect.

In contrast, according to the fragmented-framework hypothesis, the patterns of results are due to the spatial information in the study sentences. So, from this perspective, altering the additional phrase in the study sentences to include nonspatial information would not influence the structure of the situation models. More specifically, the pattern of results should differ from that observed in Experiments 1 and 2 and should more closely resemble that observed in other experiments (e.g., Radvansky, 1999a). Specifically, there should be a fan effect in the multiple-locations condition, but not in the single-location condition.

Like in Experiment 1 and in the standard-study group of Experiment 2, the participants in Experiment 3 studied the sentences one at a time. Also, like in Experiment 2, the multiple variations of the nonstudied probes were used to decrease the possibility of the participants' focusing on some aspects of the probe sentences over others. 


\section{Method}

Participants. Twenty-three people were recruited from the participant pool at the University of Notre Dame and were given partial course credit for their participation.

Materials and Procedure. The most salient difference between the methods for Experiments 1 and 2 and that for Experiment 3 was a change in the additional phrases used. Specifically, instead of spatial directions, the phrases "is being repaired," "is being cleaned," and "is being removed" were used instead. Beyond this, the materials and procedure were similar to those in Experiments 1 and 2, except that (1) the complete probes were always presented for the 432 -item recognition test, (2) the 18 study sentences were always presented one at a time, and (3) the negative probes were always created by altering the object, relation, and location terms. Like in Experiment 2, the negative probes - the three versions of each sentence negative type-occurred equally often across the recognition test. Also like in Experiments 1 and 2, half of the recognition probes were positive, and half were negative probes.

\section{Results}

Learning. The participants took 3-11 study-test cycles memorize the study sentences $(M=6.6, S E=0.51)$.

Recognition. The response time and error rate data are reported in Table 3 . The trimming procedure eliminated $1.7 \%$ of the response time data. The response time and error rate data were submitted to 2 (truth value: true vs. false) $\times 2$ (location: single location vs. multiple locations) $\times 3$ (fan) repeated measures ANOVAs.

For the response times, there was a main effect of truth value $\left[F(1,22)=5.76, M S_{\mathrm{e}}=71,760, p=.03\right]$, with the participants responding faster to true probes $(1,401 \mathrm{msec})$ than to false probes $(1,478 \mathrm{msec})$. Also, there was a significant main effect of location $\left[F(1,22)=12.49, M S_{\mathrm{e}}=\right.$ $116,991, p=.002]$ and fan $\left[F(2,44)=15.23, M S_{\mathrm{e}}=\right.$ $93,943, p<.001]$, as well as a significant location $\times$ fan interaction $\left[F(2,44)=9.42, M S_{\mathrm{e}}=103,060, p<.001\right]$. Simple effects tests revealed that, unlike in Experiments 1 and 2 and consistent with the fragmented-framework hypothesis, the fan effect was significant for the multiplelocations condition [Fan Level 1, $M=1,257 \mathrm{msec}$; Fan Level 2, $M=1,605 \mathrm{msec}$; Fan Level 3, $M=1,676 \mathrm{msec}$; $\left.F(2,44)=27.00, M S_{\mathrm{e}}=273,208, p<.001\right]$, but not for the single-location condition [Fan Level 1, $M=$ 1,335 msec; Fan Level 2, $M=1,416$ msec; Fan Level 3, $M=1,350 \mathrm{msec} ; F(2,44)=1.04, M S_{\mathrm{e}}=81,463, p=$ .36]. No other interactions were significant (all $F_{\mathrm{s}}<1$ ).

The participants made an average of $7 \%$ errors. An analysis of the error rate data revealed a significant main effect of truth value $\left[F(1,22)=6.44, M S_{\mathrm{e}}=.003, p=.02\right]$, with the participants making fewer errors following the true probes $(M=.06)$ than following the false probes $(M=.08)$. There was also a significant main effect of location $[F(1,22)=$
7.64, $\left.M S_{\mathrm{e}}=.010, p=.01\right]$, with the participants making fewer errors in response to single-location $(M=.05)$ than to multiple-locations probes $(M=.08)$. Finally, there was a significant effect of fan $\left[F(2,44)=7.04, M S_{\mathrm{e}}=.005\right.$, $p=.002]$, with the participants making more errors with increased numbers of associations (Fan Level 1, $M=.05$; Fan Level 2, $M=.06 ;$ Fan Level 3, $M=.09$ ). None of the interactions were significant (all $F_{\mathrm{S}} \leq 1.2$ ).

\section{Discussion}

Like in Experiments 1 and 2, there was a differential interference effect, consistent with an organization by spatial location. However, in contrast to those two experiments, there was no significant interference effect for the single-location condition. So, consistent with the fragmented-framework hypothesis and inconsistent with the sentence-complexity hypothesis, interference was not observed in this condition, because the additional information in the sentences was not spatial and, so, did not create sublocations in the situation model, as was the case in Experiments 1 and 2. So, this pattern of results is inconsistent with a sentence-complexity hypothesis, which suggests that the addition phrase would have produced results in Experiment 3 that would have shown a single-location interference effect like in Experiments 1 and 2.

\section{GENERAL DISCUSSION}

Across a wide range of domains, spatial framework and relational information has been shown to have meaningful impacts on cognition (e.g., Huttenlocher et al., 1991; Kemmerer, 2006; Kosslyn, 1987; Levinson, 2003; Wyer \& Radvansky, 1999). In general, spatial framework information provides boundaries for an event. In comparison, spatial relations provide information about the relative orientations of entities within that region. The aim of the present study was to assess the degree to which these different types of information would influence the organization of situation models. More specifically, this work compared a single-framework hypothesis, which states that the direction terms only provide information about the internal structure of a situation model and would not impact retrieval, and a fragmented-framework hypothesis, which states that direction terms serve to identify subregions within the larger framework, which can then interfere with one another during retrieval. This is consistent with recent neuroimaging work that shows that both of these types of spatial knowledge use similar neural networks but with different emphases (Martin, Houssemand, Schiltz, Burnod, \& Alexandre, 2008).

Table 3

Response Times (RTs, in Milliseconds) and Error Rates (Err) for Experiment 3

\begin{tabular}{|c|c|c|c|c|c|c|c|c|c|c|c|c|}
\hline & \multicolumn{6}{|c|}{ Single Location } & \multicolumn{6}{|c|}{ Multiple Locations } \\
\hline & \multicolumn{2}{|c|}{1} & \multicolumn{2}{|c|}{2} & \multicolumn{2}{|c|}{3} & \multicolumn{2}{|c|}{1} & \multicolumn{2}{|c|}{2} & \multicolumn{2}{|c|}{3} \\
\hline & RT & Err & RT & Err & RT & $\overline{E r r}$ & RT & Err & RT & Err & RT & Err \\
\hline True & 1,280 & .03 & 1,412 & .04 & 1,344 & .06 & 1,209 & .06 & 1,492 & .07 & 1,668 & .09 \\
\hline False & 1,389 & .05 & 1,419 & .04 & 1,356 & .08 & 1,304 & .07 & 1,718 & .08 & 1,684 & .13 \\
\hline
\end{tabular}


In both experiments, we used a fan-effect paradigm (Anderson, 1974) in which the integration of information into location-based situation models has been shown to eliminate retrieval interference (Radvansky, 1999a). In Experiments 1 and 2, fan-effect interference was reliably observed in the single-location condition, in which several objects were described as being in a common location. This suggests that the different regions defined by the direction terms served as subframeworks within the larger location and that these competed with one another during retrieval, thereby producing interference. As such, this pattern of data is more consistent with a fragmentedframework than with a single-framework hypothesis.

That said, it should also be noted that there were significant interactions of fan with location (single vs. multiple locations). So, although there was interference in the single-location condition, it was not as great as in the multiple-location condition. Thus, there is some benefit to having multiple objects in a single location. There are some scaling issues here, with larger locations producing greater interference relative to the smaller directiondefined locations. There is likely a hierarchy of locationbased situation models that can be derived from these different types of locations.

This pattern of data does not appear to be a consequence of task demands at retrieval. The data were relatively unchanged when the direction terms were omitted from the memory probes (Experiment 1) or when the need to use the direction terms during retrieval was emphasized, via the way the negative probes were created (Experiment 2). Thus, the interference that is observed is most likely due to how the information was organized and structured during memorization.

Moreover, the resulting mental representations do not appear to be influenced by working memory demands during memorization. In Experiment 2, working memory load was lightened by presenting all of the facts that referred to a common location together. However, this had no meaningful impact on the pattern of data. Also, in Experiment 3, when the additional information in the study sentences was not spatial, no single-location interference effect was observed, reinforcing the idea that what was observed in the first two experiments is a spatial effect. As such, this provides stronger evidence that the organization of the information was guided more by the use of the content of the facts than by some particular aspect of the methodology.

Finally, although fan effects were consistently observed in both the single-location and multiple-locations conditions in Experiments 1 and 2, it was also always reliably larger for the multiple-locations condition. This demonstrates that, although spatial framework and relation information can both be used to segregate information and both produce interference at retrieval, they are not equivalent. The interference produced by different locations is markedly greater than that produced by different subregions within a larger location. Spatial framework information has a more dramatic impact than spatial relation information. This supports other lines of research showing that the different types of information are treated qualitatively differently by cognition (e.g., Kemmerer, 2006; Kosslyn, 1987; Levinson, 2003). Spatial framework information more clearly defines separate events. In comparison, spatial relation information only serves to more finely articulate components within that larger region.

In summary, spatial frameworks and relations were both used to organize information in situation models, which then led to interference during retrieval. Thus, both types of spatial information can impact the ease of retrieval. However, spatial frameworks had a greater impact than spatial relations. This is further support for the complexity with which different qualities of spatial information present themselves to spatial cognition.

More broadly, these data suggest that these different qualities of spatial information - namely, framework and relational information - have a direct influence on how spatial information is organized in memory. The spatial framework appears to serve as the basis for this organization as a superordinate organization that can be used to segregate and identify separate events. When information has been stored about different locations, the information about other locations tends not to influence knowledge about those other locations. This includes not only the retrieval of memory for lists of sentences, as in the present study, but also (1) spatial memory more generally, such as what is seen in research on mental maps showing that region information has a profound influence on memory for spatial knowledge (e.g., Friedman \& Brown, 2000; McNamara, 1986; Stevens \& Coupe, 1978); (2) language comprehension, such as the increased difficulty in reading when a shift occurs from one spatial framework to another in a narrative text (e.g., Zwaan et al., 1998); or (3) even memory for prior knowledge when a person moves from one room to another (e.g., Radvansky \& Copeland, 2006c).

Spatial relations also have an influence on the organization of knowledge that shares some characteristics with spatial frameworks but also exhibits some important differences as well. Specifically, spatial relations provide information about the subordinate organization within the context of a single event. As such, because all of the information is part of the same larger structure, the mental organization is not as segregated as it is when different spatial frameworks are involved. These sublocations appear to direct mental attention, in some way, to part of the framework rather than to the others. So, when information is assessed about one subregion, related information from other subregions becomes a source of interference, and cognition is compromised accordingly. In the present study, this took the form of the presence of retrieval interference, which has been absent in prior studies that did not include such spatial relations. This suggests that spatial relations generally serve to foreground certain parts of the environment but still leave other parts in a heightened state of availability by which they can still influence the current stream of thought.

In conclusion, both spatial framework and spatial relation information influence the mental organization of events, as was made evident by the present assessment of the availability of information stored in situation models 
in long-term memory. Although spatial framework information had a stronger influence on the structure of this organization, spatial relation information did further subdivide the larger spatial framework in a way that served to direct attention to and to foreground certain parts of a given framework relative to other parts, which could then become a source of retrieval interference.

\section{AUTHOR NOTE}

This research was supported by a Faculty Research Grant from the Graduate School at the University of Notre Dame to the author. The ideas presented here were initially developed while the author was supported by NIMH National Research Service Award No. MH14257, while a postdoctoral trainee in the Quantitative Methods Program of the Department of Psychology, University of Illinois at Urbana-Champaign. I thank Laura Bodien, Jackie Curiel, Sharron Francis, Brittany Love, Mark Lutz, and Dan Olsen for their assistance in collecting the data. Correspondence should be addressed to G. A. Radvansky, Department of Psychology, University of Notre Dame, Notre Dame, ID 46556 (e-mail: gradvans@nd.edu).

\section{REFERENCES}

ANDERSON, J.R. (1974). Retrieval of propositional information from longterm memory. Cognitive Psychology, 6, 451-474. doi:10.1016/0010 $-0285(74) 90021-8$

Bransford, J. D., Barclay, J. R., \& Franks, J. J. (1972). Sentence memory: A constructive versus interpretive approach. Cognitive Psychology, 3, 193-209. doi:10.1016/0010-0285(72)90003-5

Bunting, M. F., Conway, A. R. A., \& Heitz, R. P. (2004). Individual differences in the fan effect and working memory capacity. Journal of Memory \& Language, 51, 604-622. doi:10.1016/j.jml.2004.07.007

CANTOR, J., \& ENGLE, R. W. (1993). Working memory capacity as longterm memory activation: An individual differences approach. Journal of Experimental Psychology: Learning, Memory, \& Cognition, 19, 1101-1114. doi:10.1037/0278-7393.19.5.1101

Franklin, N., \& TVersky, B. (1990). Searching imagined environments. Journal of Experimental Psychology: General, 119, 63-76. doi:10.1037/0096-3445.119.1.63

Friedman, A., \& Brown, N. R. (2000). Reasoning about geography. Journal of Experimental Psychology: General, 129, 193-219. doi:10.1037/0096-3445.129.2.193

Garnham, A. (1981). Mental models as representations of text. Memory \& Cognition, 9, 560-565.

Glenberg, A. M., Meyer, M., \& Lindem, K. (1987). Mental models contribute to foregrounding during text comprehension. Journal of Memory \& Language, 26, 69-83. doi:10.1016/0749-596X(87) 90063-5

Huttenlocher, J., Hedges, L. V., \& Duncan, S. (1991). Categories and particulars: Prototype effects in estimating spatial location. Psychological Review, 98, 352-376. doi:10.1037/0033-295X.98.3.352

JoHnSON-LAIRD, P. N. (1983). Mental models. Cambridge, MA: Harvard University Press.

Kemmerer, D. (2006). The semantics of space: Integrating linguistic typology and cognitive neuroscience. Neuropsychologia, 44, 16071621. doi:10.1016/j.neuropsychologia.2006.01.025

KossLyn, S. M. (1987). Seeing and imagining in the cerebral hemispheres: A computational approach. Psychological Review, 94, 148175. doi: $10.1037 / 0033-295 X .94 .2 .148$

Levinson, S. C. (2003). Space in language and cognition. Cambridge: Cambridge University Press.

Martin, R., Houssemand, C., Schiltz, C., Burnod, Y., \& AlexanDRE, F. (2008). Is there continuity between categorical and coordinate spatial relations coding? Evidence from a grid/no-grid working memory paradigm. Neuropsychologia, 46, 576-594. doi:10.1016/j .neuropsychologia.2007.10.010

McNamara, T. P. (1986). Mental representations of spatial relations. Cognitive Psychology, 18, 87-121. doi:10.1016/0010-0285(86)90016-2

MoESER, S. D. (1979). The role of experimental design in investigations of the fan effect. Journal of Experimental Psychology: Human Learning \& Memory, 5, 125-134.
Morrow, D. G., Greenspan, S. L., \& Bower, G. H. (1987). Accessibility and situation models in narrative comprehension. Journal of Memory \& Language, 26, 165-187. doi:10.1016/0749-596X(87)90122-7

Myers, J. L., O’Brien, E. J., Balota, D. A., \& Toyofuku, M. L. (1984). Memory search without interference: The role of integration. Cognitive Psychology, 16, 217-242. doi:10.1016/0010-0285(84)90008-2

RADVANSKY, G. A. (1998). The organization of information retrieved from situation models. Psychonomic Bulletin \& Review, 5, 283-289.

RadVAnsky, G. A. (1999a). The fan effect: A tale of two theories. Journal of Experimental Psychology: General, 128, 198-206. doi:10.1037/0096-3445.128.2.198

RADVANSKY, G. A. (1999b). Memory retrieval and suppression: The inhibition of situation models. Journal of Experimental Psychology: General, 128, 563-579. doi:10.1037/0096-3445.128.4.563

RADVANSKY, G. A. (2005). Situation models, propositions, and the fan effect. Psychonomic Bulletin \& Review, 12, 478-483.

Radvansky, G. A., \& Copeland, D. E. (2000). Functionality and spatial relations in situation models. Memory \& Cognition, 28, 987-992.

Radvansky, G. A., \& Copeland, D. E. (2006a). Memory retrieval and interference: Working memory issues. Journal of Memory \& Language, 55, 33-46. doi:10.1016/j.jml.2006.02.001

Radvansky, G. A., \& Copeland, D. E. (2006b). Situation models and retrieval interference: Pictures and words. Memory, 14, 614-623. doi:10.1080/09658210600647714

RadVAnSky, G. A., \& Copeland, D. E. (2006c). Walking through doorways causes forgetting. Memory \& Cognition, 34, 1150-1156.

RadVansky, G. A., Spieler, D. S., \& ZaCKs, R. T. (1993). Mental model organizations. Journal of Experimental Psychology: Learning, Memory, \& Cognition, 19, 95-114. doi:10.1037/0278-7393.19.1.95

Radvansky, G. A., Wyer, R. S., JR., Curiel, J. M., \& Lutz, M. F. (1997). Situation models and abstract ownership relations. Journal of Experimental Psychology: Learning, Memory, \& Cognition, 23, 1233-1246. doi:10.1037/0278-7393.23.5.1233

RadVAnsky, G. A., \& ZaCKs, R. T. (1991). Mental models and the fan effect. Journal of Experimental Psychology: Learning, Memory, \& Cognition, 17, 940-953. doi:10.1037/0278-7393.17.5.940

RADVANSKY, G. A., \& ZACKS, R. T. (1997). The retrieval of situationspecific information. In M. A. Conway (Ed.), Cognitive models of memory (pp. 173-213). Cambridge, MA: MIT Press.

Radvansky, G. A., Zacks, R. T., \& HASHER, L. (1996). Fact retrieval in younger and older adults: The role of mental models. Psychology \& Aging, 11, 258-271. doi:10.1037/0882-7974.11.2.258

RADVANSKY, G. A., ZACKs, R. T., \& HASHer, L. (2005). Age and inhibition: The retrieval of situation models. Journals of Gerontology, $60 \mathrm{~B}$, P276-P278

Radvansky, G. A., Zwaan, R. A., Federico, T., \& Franklin, N. (1998). Retrieval from temporally organized situation models. Journal of Experimental Psychology: Learning, Memory, \& Cognition, 24, 1224-1237. doi: $10.1037 / 0278-7393.24 .5 .1224$

REDER, L. M., \& ANDERSON, J. R. (1980). A partial resolution of the paradox of interference: The role of integrating knowledge. Cognitive Psychology, 12, 447-472. doi:10.1016/0010-0285(80)90016-X

Smith, E. E., Adams, N., \& Schorr, D. (1978). Fact retrieval and the paradox of interference. Cognitive Psychology, 10, 438-464. doi:10.1016/0010-0285(78)90007-5

Stevens, A., \& Coupe, P. (1978). Distortions in judged spatial relations. Cognitive Psychology, 10, 422-437. doi:10.1016/0010 $-0285(78) 90006-3$

VAN DisK, T. A., \& KinTsCH, W. (1983). Strategies in discourse comprehension. New York: Academic Press.

WYER, R. S., \& RADVANSKY, G. A. (1999). The comprehension and validation of social information. Psychological Review, 106, 89-118. doi:10.1037/0033-295X.106.1.89

ZwaAn, R. A. (1999). Situation models: The mental leap into imagined worlds. Current Directions in Psychological Science, 8, 15-18. doi:10.1111/1467-8721.00004

ZwaAn, R. A., \& Radvansky, G. A. (1998). Situation models in language comprehension and memory. Psychological Bulletin, 123, 162185. doi:10.1037/0033-2909.123.2.162

Zwaan, R. A., Radvansky, G. A., Hilliard, A. E., \& Curiel, J. M. (1998). Constructing multidimensional situation models during reading. Scientific Studies of Reading, 2, 199-220. doi:10.1207/ s1532799xssr0203_2 
APPENDIX

Objects and Locations Used to Generate the Sentences Used in the Experiments

\begin{tabular}{ll}
\hline \multicolumn{1}{c}{ Objects } & \multicolumn{1}{c}{ Locations } \\
\hline broken window & (movie) theater \\
waste basket & (public) library \\
potted palm & city hall \\
drinking fountain & (grand) hotel \\
(large) poster & airport \\
pay phone & office building \\
(oak counter) shelf & high school \\
(wall) clock & cocktail lounge \\
bulletin board & laundromat \\
closet door & ice cream parlor \\
coat rack & car dealership \\
fire extinguisher & barber shop \\
\hline
\end{tabular}

Note-The words in parentheses were used in Experiment 1, but not Experiments 2 and 3 .

(Manuscript received November 30, 2008; revision accepted for publication March 31, 2009.) 